\title{
Distrofia muscular de Duchenne em hospital pediátrico de uma cidade no estado do Pará
}

\author{
Duchenne muscular dystrophy in a pediatric hospital in a city in the state of Pará
}

Distrofia muscular de Duchenne en un hospital pediátrico en una ciudad del estado de Pará

Marina Geórgia Cruz Keuffer ${ }^{1 *}$, Emanuelle Avís de Oliveira ${ }^{1}$, Ara Rubia Costa Gonçalves ${ }^{1}$, Amanda Soares Peixoto², Érika de Oliveira Santos ${ }^{1}$, Raina Caroline Batista Quaresma².

\begin{abstract}
RESUMO
Objetivo: Relatar o caso de paciente atendido no ambulatório de neurologia de um hospital pediátrico de uma cidade no estado do Pará com o diagnóstico de Distrofia Muscular de Duchenne, durante os anos de 2018 e 2019. Detalhamento de caso: Paciente do sexo masculino, 9 anos de idade, procedente de uma cidade no interior do estado do Pará com história de dificuldade para manter-se em ortostase e atraso no desenvolvimento neuropsicomotor, evoluiu com piora progressiva. Na primeira consulta observado marcha anserina ainda independente, sinal de Gowers positivo e pseudo-hipertrofia de panturrilhas. Apresentou diagnóstico confirmado de Distrofia Muscular de Duchenne através de sequenciamento genético, iniciou o tratamento medicamentoso com prednisolona e, em seguida, foi associado o atalureno. Considerações finais: A disponibilidade de exames laboratoriais e o fechamento do diagnóstico contribuíram para a orientação do tratamento específico, no entanto, a procura por ajuda médica tardia, a escassez de profissionais de saúde na atenção básica da região, a irregularidade do tratamento e o abandono da reabilitação agravaram o quadro do paciente.
\end{abstract}

Palavras-chave: Distrofia, Duchenne, Pediatria.

\begin{abstract}
Objective: To describe a case report of a patient undergoing follow-up at the neurology outpatient clinic in a pediatric hospital in a city in the state of Pará with the diagnosis of Duchenne Muscular Dystrophy during the years 2018 and 2019. Case details: Male patient, 9 years old, coming from a city in the interior of the state of Pará with a history of difficulty in maintaining orthostasis and delayed neuropsychomotor development, progressing with progressive worsening. During the first consultation, an independent anserine gait, a positive Gowers sign and pseudohypertrophy of the calves were observed. He presented a confirmed diagnosis of Duchenne Muscular Dystrophy through genetic sequencing, started treatment with prednisolone and then associated with ataluren. Final considerations: The availability of laboratory tests and the closing of the diagnosis helped at the beginning of the treatment, providing an initial improvement in motor symptoms, however, the search for late medical help, the shortage of basic health professionals in basic health care in the region, the irregularity of the treatment and abandonment of rehabilitation worsened the patient's condition.
\end{abstract}

Keywords: Dystrophy, Duchenne, Pediatrics.

\section{RESUMEN}

Objetivo: Describir el caso de un paciente atendido en el ambulatorio de neurología en un hospital pediátrico en una ciudad del estado de Pará con el diagnostico de Distrofia Muscular de Duchenne, durante los años

1 Fundação Santa Casa de Misericórdia do Pará, Belém - PA. *E-mail: mkeuffer@hotmail.com

2 Hospital Bettina Ferro de Souza da Universidade Federal do Pará, Belém - PA.

SUBMETIDO EM: 2/2021

ACEITO EM: 5/2021

PUBLICADO EM: 6/2021 
2018 y 2019. Detalles del caso: Sexo masculino, 9 años de edad, procedente de una ciudad del interior del estado de Pará con historia de dificultad para mantener la ortostasis y retraso en el desarrollo neuropsicomotor, progresó con empeoramiento progresivo. En la primera consulta se observó marcha anserina independiente, señal de Gowers positivo y pseudohipertrofia de las pantorrillas. Tenía un diagnóstico confirmado de Distrofia Muscular de Duchenne mediante secuenciación genética, inició tratamiento farmacológico con prednisolona y luego se asoció ataluren. Consideraciones finales: La disponibilidad de pruebas de laboratorio y el cierre del diagnóstico contribuyó a la orientación del tratamiento específico, sin embargo, la búsqueda de ayuda médica tardía, la escasez de profesionales de la salud en atención primaria en la región, la irregularidad del tratamiento y el abandono. la rehabilitación agravó el estado del paciente.

Palabras clave: Distrofia, Duchenne, Pediatría.

\section{INTRODUÇÃO}

A Distrofia Muscular de Duchenne (DMD) é uma doença genética neuromuscular rara, de caráter recessivo ligado ao cromossomo $X$ que afeta 1 a cada 3500 crianças nascidas do sexo masculino predominantemente, de forma irreversível, sendo a forma mais comum de distrofia muscular. Pacientes do sexo feminino desenvolvem a forma assintomática da doença (NEGRONI E, et al., 2015; SOUZA IE, et al., 2015).

A doença ocorre devido mutações na estrutura do gene que codifica a proteína distrofina, localizado no cromossomo Xp21, que resultam em uma deficiência da mesma. A distrofina é responsável pela manutenção da integridade das fibras musculares e pela mediação das cascatas de sinalização citoplasmática e das funções musculares. Sua deficiência resulta em degeneração generalizada do sistema muscular (NEGRONI E, et al., 2015; STREHLE EM e STRAUB V, 2015).

A DMD geralmente se apresenta na primeira infância com atrasos nos marcos motores, incluindo atrasos para andar sozinho e levantar da posição supina. A fraqueza muscular proximal causa uma marcha gingada e dificuldade para subir escadas, correr, pular e levantar-se da posição agachada. A doença é rapidamente progressiva, simétrica e bilateral, e a deambulação geralmente é perdida entre os 10-12 anos de idade, tornando a criança dependente de cadeira de rodas (DONEGÁ RA e RUZZON DV, 2014).

A cardiomiopatia, principalmente a disfunção ventricular, ocorre em quase todos os indivíduos com DMD após os 18 anos, caracterizado por elevação da enzima creatinofosfoquinase (CK) sérica (SANTOS MA, et al., 2010). Poucos sobrevivem além da terceira década, com complicações respiratórias e cardíacas, sendo estas causas comuns de morte (DARRAS MD, et al., 2018; NEGRONI E, et al., 2015).

O diagnóstico precoce é importante para que as opções de tratamento estejam disponíveis o mais cedo possível. Deve ser pensado, inicialmente, mediante a presença das manifestações clínicas ou de achados como função muscular anormal, aumento da creatinoquinase sérica ou de transaminases. A confirmação poderá ser feita mediante o teste para mutação de DMD em amostra de sangue ou saliva, através do sequenciamento do gene da distrofina ou de biópsia muscular. Apesar desta última ser um exame de confirmação diagnóstica, é mandatório realizar teste genético. Os testes geralmente utilizados para a sua detecção são o multiplex PCR, multiplex ligation dependente probe amplification, single-condition amplification / internal primer and multiplex amplifiable probe hybridisation (BALTAZAR GF, et al., 2019).

No momento ainda não existe cura para DMD. Algumas terapias são utilizadas como fisioterapia, hidroterapia e terapia medicamentosa com fármaco de corticoides (QUINLIVAN R, 2014; STREHLE EM e STRAUB V, 2015; BOAS HJ e MOREIRA DO, 2020). Atualmente já estão disponíveis no mercado novos tratamentos modificadores de doença para a DMD, entre os quais se encontra o atalureno. É um oligonucleotídeononsense oralmente disponível que se liga ao RNAm, impedindo a interrupção precoce da leitura do códon pelo RNAm durante a transcrição da proteína distrofina. Somente tem efeito em pacientes com mutação "nonsense", que ocorre em aproximadamente 15\% dos casos (ABDALLAH OA, et al., 2018).

O atalureno, na dose de $40 \mathrm{mg} / \mathrm{kg} / \mathrm{dia}$, é a primeira terapia modificadora de doença aprovada pela Agência Nacional de Vigilância Sanitária (ANVISA), através da Resolução-RE Número 1.01, de 25 de abril de 2019, 
que visa o tratamento de pacientes a partir de 2 anos de idade, principalmente na fase de transição ambulatorial, com mutações nonsense (CAMPBELL C, et al., 2020).

Portanto, objetiva-se alertar aos pediatras gerais sobre a possibilidade de considerar entre os diagnósticos diferenciais de uma criança com atraso no desenvolvimento motor a possibilidade da distrofia muscular de Duchenne, contribuindo dessa forma para o diagnóstico precoce, principalmente por já se encontrarem disponíveis tratamentos que são modificadores de doença, e para incentivar novas publicações.

\section{DETALHAMENTO DO CASO}

Paciente F.H.T.S, 9 anos de idade, sexo masculino, pardo, procedente de Viseu - Pará, foi acompanhado no ambulatório de neurologia de um hospital pediátrico de uma cidade no estado do Pará desde julho de 2018 devido dificuldade para manter-se em ortostase e atraso no desenvolvimento neuropsicomotor. Foi atendido inicialmente em outro serviço em Ananindeua - Pará e posteriormente encaminhado para o serviço de referência da Fundação Santa Casa de Misericórdia do Pará, onde deu continuidade no seu acompanhamento.

É o primeiro filho de pais não consanguíneos, nasceu de parto vaginal em casa sem intercorrências durante a gestação e no período neonatal. A mãe negou abortos prévios. Apresentou cartão vacinal desatualizado. Sobre os antecedentes familiares, dois tios maternos já haviam falecido com quadro semelhante ao da criança. Genitora informou que o menor sempre teve dificuldades na marcha, começou a andar a partir dos 2 anos de idade e evoluiu com piora progressiva no aspecto motor (aos 3 anos locomoviase somente com apoio). Além da dificuldade motora, a criança apresentou atraso na linguagem e dispneia aos esforços.

Durante o exame físico na primeira consulta, observou-se como dados positivos: marcha anserina (afastamento das pernas, lordose exagerada e movimento oscilatório da bacia) ainda independente, sinal de Gowers positivo (o levantar miopático, no qual para levantar-se do chão o paciente usa as mãos para escalar as próprias pernas) e pseudo-hipertrofia de panturrilhas bem evidentes. Realizou exames laboratoriais em julho de 2018 que evidenciaram: Aldolase: 79,5 U/L (Valor de referência: 1,2-8,8), CKMB: 273 U/L (Valor de referência: <24) e CPK: 4959 U/L (Valor de referência: até 171).

$\mathrm{Na}$ primeira consulta a criança foi referenciada para acompanhamento com equipe multiprofissional (fonoaudiologia, fisioterapia, terapia ocupacional e psicologia), com pneumopediatria, ortopedia, endocrinopediatria, cardiopediatria e para atualização vacinal no Centro de Referência para Imunobiológicos Especiais (CRIE). Foram solicitados exames laboratoriais gerais, sequenciamento genético para pesquisa de Distrofia Muscular de Duchenne, ecocardiograma, radiografia de tórax e eletrocardiograma.

$\mathrm{Na}$ consulta subsequente paciente retornou ainda sem o resultado do sequenciamento genético para Distrofia Muscular de Duchenne, porém com o resultado de exames laboratoriais: $\mathrm{Hb}$ 13,0; $\mathrm{Ht} 41,4 \%$; Leucócitos: 6805; Plaquetas: 212.900; Proteínas: 5,90; Magnésio: 2,0; Amilase: 39,0; Sódio: 143,0; Potássio: 4,2; Cloro: 104; TGO: 251; TGP: 383; Fosfatase Alcalina: 115,0; Gama GT: 9,0; Creatinina: 0,50; Uréia 18,0; Glicose: 86,0. Radiografia de tórax sem alterações. Foi encaminhado para o ambulatório de gastropediatria devido sintomas de refluxo gastroesofágico e, posteriormente, a mesma solicitou avaliação da hepatologia devido alteração da função hepática.

Apresentou diagnóstico confirmado de Distrofia Muscular de Duchenne por Sequenciamento do Gene DMN (NM_004006). [Interpretação descrita no exame: Foi encontrada a variante c.1684C>T p.(Gln562Ter) no exon 14 do gene DMD, em hemizigose (mutação "nonsense"). Essa variante substitui uma Glutamina por um códon de parada no códon 562 da proteína traduzida, gerando consequentemente uma proteína truncada. O acúmulo de evidências permite a sua classificação como patogênica.

Em decorrência de ser uma patologia de herança ligada ao $\mathrm{X}$, esse achado confirma o diagnóstico clínico de Duchenne em indivíduos do sexo masculino. O resultado do teste foi entregue para a família somente em janeiro de 2019, quando iniciou corticoterapia com prednisolona $3 \mathrm{mg} / \mathrm{ml}(1 \mathrm{mg} / \mathrm{kg} / \mathrm{dia}) 7 \mathrm{ml} 1 \mathrm{vez}$ ao dia pela manhã e encaminhado para reabilitação. 
Tabela 1 - Valores de referência dos exames laboratoriais segundo a idade e sexo do paciente em questão.

\begin{tabular}{cc}
\hline Exames Laboratoriais & Valores de Referência (VR) \\
\hline Aldolase & $1,2-8,8 \mathrm{U} / \mathrm{L}$ \\
Creatinofosfoquinase MB (CKMB) & $<24,0 \mathrm{U} / \mathrm{L}$ \\
Creatinofosfoquinase (CPK) & Até $171,0 \mathrm{U} / \mathrm{L}$ \\
Hemoglobina & $11,5-13,5 \mathrm{~g} / \mathrm{dL}$ \\
Hematócrito & $35-40 \%$ \\
Leucócitos totais & $4.500-13.500 \mathrm{céls} / \mathrm{mm} 3$ \\
Glicemia em jejum & $60-100 \mathrm{mg} / \mathrm{dL}$ \\
Proteínas totais & $6,0-8,0 \mathrm{~g} / \mathrm{dL}$ \\
Amilase & $60-160 \mathrm{U} / \mathrm{dL}$ \\
Lipase & $10-37 \mathrm{U} / \mathrm{L}$ \\
Transaminase glutâmico-oxalacética (TGO) & $10-41 \mathrm{U} / \mathrm{L}$ \\
Transaminase glutâmico-pirúvica (TGP) & $24-49 \mathrm{U} / \mathrm{L}$ \\
Fosfatase alcalina & $75-390 \mathrm{U} / \mathrm{L}$ \\
Gama GT & $3-22 \mathrm{U} / \mathrm{L}$ \\
Uréia & $8-36 \mathrm{mg} / \mathrm{dL}$ \\
Creatinina & $0,57-0,89 \mathrm{mg} / \mathrm{dL}$ \\
Magnésio & $1,6-2,6 \mathrm{mg} / \mathrm{dL}$ \\
Sódio & $135-148 \mathrm{mEq} / \mathrm{L}$ \\
Potássio & $3,5-5,5 \mathrm{mEq} / \mathrm{L}$ \\
Cloreto & $98-109 \mathrm{mEq} / \mathrm{L}$ \\
\hline
\end{tabular}

Fonte: Keuffer MG, et al., 2021.

Retornou em consulta posterior fazendo uso contínuo de corticoterapia com boa aceitação e realizando fisioterapia semanalmente, na cidade de origem. Ao exame físico, observou-se marcha ainda independente, porém com menor dificuldade no levantar, demonstrando maior agilidade. Foi entregue para os responsáveis laudo com a receita para o atalureno e reduzida dose da prednisolona $3 \mathrm{mg} / \mathrm{ml}(0,75 \mathrm{mg} / \mathrm{kg} / \mathrm{dia}) 5,5 \mathrm{ml} 1 \mathrm{vez}$ ao dia pela manhã.

No atendimento seguinte retornou mantendo uso constante da corticoterapia, porém sem ainda ter iniciado o atalureno, naquela ocasião estava sem fisioterapia. Evoluiu com evidente piora no aspecto motor e com maior dificuldade em realizar os movimentos (mantinha-se em pé e deambulava com apoio externo nos objetos, sinal de Gowers mais prolongado). Foram coletados exames laboratoriais, reforçado procurar reabilitação e modificado esquema da prednisolona sem mudança de dose (passando para uso diário por 10 dias consecutivos, com "stop" de 10 dias, esquema de tratamento alternado). Entregue novamente laudo e receita para a aquisição do atalureno e solicitado retorno em 30 dias. Paciente não retornou para atendimento após esta data no ano de 2019.

Em acompanhamento ambulatorial na FSCMPA com a equipe multiprofissional, endocrinopediatria, gastropediatria, hepatologia e pneumopediatria, além de avaliação com a ortopedia em ambulatório externo. Recebeu os diagnósticos de síndrome aspirativa por provável Doença do Refluxo Gastroesofágico, síndrome do respirador oral, transtorno respiratório do sono (presença de roncos habituais) e asma controlada. Ainda aguardava consulta com a cardiologia pediátrica na FSCMPA.

\section{DISCUSSÃO}

O diagnóstico precoce da Distrofia Muscular de Duchenne é um fator importante para evitar o aumento do grau de avanço da doença, uma vez que o tratamento com corticoides tem se mostrado eficaz no retardo desta progressão, além da iniciação da fisioterapia para minimizar contraturas que dificultam a mobilidade. Os primeiros sintomas surgem até o terceiro ano de idade, no entanto o diagnóstico no início da infância é um desafio maior para famílias com acesso restrito à saúde (MOXLEY RT, et al., 2005; CAROMANO FA, 1999). 
No presente relato notou-se que desde a primeira consulta os sinais clássicos da doença já estavam bem evidentes no paciente, provavelmente devido ao momento tardio da procura por ajuda médica. Este fato pode ter ocorrido devido a cidade de origem da família, Viseu, ser localizada no nordeste paraense e com pouco mais de 60.000 habitantes (IBGE, 2020). Os prestadores de cuidados primários são, em geral, o primeiro ponto de contato com a saúde dos portadores da DMD, e o estado do Pará é o estado com menor cobertura assistencial primária no norte do país, com cobertura de apenas $50,5 \%$ do território. Esta situação dificulta o acesso ao atendimento médico adequado para a população residente no interior do estado (GARNELO L, et al., 2018; NORITZ G, et al., 2018).

Os profissionais da assistência básica devem ser encorajados a se familiarizar com doenças raras como a DMD para monitorar, reconhecer e agir, tratando dos sintomas neuromusculares iniciais, assim como de cuidados primários tradicionais como imunização, orientação prévia e triagem de doenças específicas, além de exames de rotina. Desta forma, com o devido treinamento dos profissionais, muitos casos podem ser diagnosticados precocemente para que o paciente receba o tratamento correto. (NORITZ G, et al., 2018).

Devido as alterações na distrofina, pacientes com DMD apresentam altos níveis de TGO (transaminase glutâmico-oxalacética) e TGP (transaminase glutâmico-pirúvica), fato que comumente induz os médicos a se concentrarem no estudo hepático, como neste estudo o paciente foi encaminhado para a hepatologia devido alterações nestas enzimas (FONSECA JG, et al., 2007).

Para um diagnóstico diferencial, o exame de creatinofosfoquinase (CPK) pode ajudar, devido ao aumento das desaminases estar diretamente relacionado à creatina quinase total nos casos de DMD (AVARIA MA, et al., 2012). Araújo AP, et al. (2017) afirma que se a suspeita clínica de DMD for apoiada por um aumento acentuado nos níveis de CPK, um teste confirmatório deve ser solicitado. Diante do resultado dos exames alterados para as três enzimas, o médico agiu corretamente ao solicitar o sequenciamento do Gene DMN (NM_004006).

O tratamento com corticoides para a DMD pode obedecer a três protocolos: (1) dose diária; (2) dose intermitente, dose diária por 10 dias, com 10 dias de interrupção do medicamento e (3) dose alta semanal por dois dias consecutivos da semana, podendo ser utilizados a prednisona, prednisolona ou deflazacort (FEDER D e LANGER AL, 2005). Neste relato, inicialmente o paciente apresentou boa aceitação à corticoterapia por dose diária, com melhora na agilidade, como esperado, e manutenção correta da fisioterapia. No entanto, ao retornar 4 meses depois, observou-se piora do quadro provavelmente devido à interrupção da reabilitação.

Para o tratamento de DMD, estudos que demonstram os benefícios da corticoterapia são publicados desde a década de 1970 (DRACHMAN DB, et al., 1974). Tais benefícios são: melhora na função motora, proporcionando marcha independente mais longa; melhor estabilização central e função do membro superior e prevenção de deformidades da coluna (LEBEL DE, et al., 2013; MATTHEWS E, et al., 2016). A terapia com glicocorticoide é recomendada para meninos com DMD na faixa etária de dois a cinco anos, de preferência na fase de platô dos déficits motores ou mesmo na fase de declínio da função motora, e para todos os meninos com mais de cinco anos, independentemente do estado funcional (BUSHBY K, et al., 2010).

O tratamento escolhido no primeiro momento foi a dose diária de $1,0 \mathrm{mg} / \mathrm{kg}$ de prednisolona e posteriormente a dose foi reduzida para $0,75 \mathrm{mg} / \mathrm{kg}$ de peso, afim de se enquadrar na dose recomendada pelo Consenso Brasileiro de Duchenne - Parte 1, modificando, assim, o esquema para dose intermitente (dose diária de $0,75 \mathrm{mg} / \mathrm{kg}$ de peso de prednisolona por 10 dias, com 10 dias de interrupção do medicamento). Este último foi escolhido na tentativa de diminuir os efeitos colaterais do corticóide, que são maior risco de descalcificação dos ossos e mais ganho de peso, enquanto que a escolha do fármaco tem por vantagem o baixo custo (ARAUJO AP, et al., 2017).

Pacientes que receberam deflazacort $(0,9 \mathrm{mg} / \mathrm{Kg})$ experimentaram magnitudes significativamente menores de declínio na função quando comparados a pacientes que foram tratados com prednisona ou prednisolona, além de apresentarem menos efeitos colaterais. A desvantagem deste fármaco é que não está disponível no sistema único de saúde e possui alto custo no mercado (MCDONALD CM, et al., 2020; TAWALBEH S, et al., 2020). 
Uma série de terapias promissoras com alvos moleculares foram desenvolvidas e algumas delas passaram de ensaios pré-clínicos para ensaios clínicos nos últimos 20 anos. O atalureno é uma droga oral que atua no nível do ribossomo, induzindo a leitura de códons de parada prematuros devido a mutações sem sentido. Os resultados da eficácia da medicação foram observados no estudo de fase $2 \mathrm{~b}$, controlado, multicêntrico, randomizado, duplo-cego (PTC124-GD-007-DMD) com 174 pacientes, a grande maioria com DMD alocados nos grupos placebo, atalureno na dose de $40 \mathrm{mg} / \mathrm{kg} / \mathrm{dia}(10,10$ e $20 \mathrm{mg} / \mathrm{kg})$ e na dose de $80 \mathrm{mg} / \mathrm{kg} / \mathrm{dia}(20,20$, $40 \mathrm{mg} / \mathrm{kg}$ ) (BUSHBY K, et al., 2014).

Ainda, os resultados da eficácia do atalureno também foram identificados no estudo de fase 3 , controlado, multicêntrico, randomizado, duplo-cego (PTC124-GD-020-DMD) com 230 pacientes com DMD em uso de corticosteroides, alocados nos grupos placebo e atalureno na dose de $40 \mathrm{mg} / \mathrm{kg} / \mathrm{dia}(10,10 \mathrm{e} 20 \mathrm{mg} / \mathrm{kg})$ (BUSHBY K, et al., 2014). O estudo de fase 3 permitiu a aprovação da medicação pela ANVISA.

Ataluren ${ }^{\circledR}$, nome comercial dado ao atalureno, recebeu autorização de comercialização condicional da Comissão Europeia para tratar pacientes ambulatoriais com DMD, com idade igual ou superior a cinco anos, com mutação sem sentido da DMD, considerando sua relação risco-benefício (EMA, 2020). Desta forma, devido a promessa dessa droga, o medicamento foi indicado ao paciente relatado. No entanto, na consulta seguinte o paciente ainda não havia iniciado e tratamento e devido ao não retorno do paciente posteriormente, não pudemos verificar se houveram melhoras no quadro após seu uso.

Portanto, enfatizou-se a importância da instituição do tratamento precoce para melhorar a qualidade de vida e reduzir a incidência de complicações de pacientes portadores da DMD. Notou-se que a procura tardia à ajuda médica e a irregularidade na frequência do seguimento podem influenciar negativamente no prognóstico da doença. Este estudo contribuiu com avanços no conhecimento científico junto à comunidade acadêmica e aos profissionais da área da saúde através de informações pertinentes sobre a DMD, reduzindo as subnotificações.

\section{REFERÊNCIAS}

1. ARAUJO AP, et al. Consenso brasileiro sobre distrofia muscular de Duchenne, parte 1, 2017. Disponível em: https://doi.org/10.1590/0004-282X20170112. Acessado em: 17 de janeiro de 2021.

2. AVARIA MA, et al. Aumento de transaminasas: Una manifestación de distrofia muscular de Duchenne. Revista Chilena de Pediatria, 2012; 83: 258-261.

3. BALTAZAR GF, et al. Distrofia muscular de Duchenne: Relato de caso e revisão bibliográfica. Revista Emescan de Estudos em Saúde, 2019; 1: 401-429.

4. BOAS HJ, MOREIRA DO. Atuação da fisioterapia na manutenção da marcha em pacientes distróficos. Revista Científica Pro Homine, 2020; 3: 26-37.

5. BRASIL, IBGE. Censo Demográfico, 2020. Disponível em www.ibge.gov.br. Acesso em: 14 de janeiro de 2021.

6. BRASIL. Manual do Ministério da Saúde, 2018; 2: 3-4. Disponível em: http: //sei.saude.gov.br/sei/controlador_externo.php?acao=documento_conferir\&acao_origem=documento_conferir\&id_or gao_acesso_externo=0\&lang=es_ES. Acessado em: 17 de janeiro de 2021.

7. BUSHBY K, et al. Ataluren treatment of patients with nonsense mutation dystrophinopathy. Muscle \& Nerve, 2014; 50: 477-487.

8. BUSHBY $\mathrm{K}$, et al. Diagnosis and management of Duchenne muscular dystrophy, part 1: Diagnosis, and pharmacological and psychosocial management. The Lancet Neurology, 2010.

9. CAMPBELL C, et al. Meta-analyses of ataluren randomized controlled trials in nonsense mutation Duchenne muscular dystrophy, 2020.

10. CAROMANO FA. Características do portador de distrofia muscular de Duchenne (DMD) - revisäo. Arquivos de Ciências da Saúde Unipar, 1999.

11. CYSNE AC, et al. Manual de exames laboratoriais da rede SUS-BH, 2016.

12. DARRAS MD, et al. Dystrophinopathies. U.S National Library of Medicine, 2018.

13. DONEGÁ RA, RUZZON DV. Distrofia muscular de Duchenne, 2014.

14. DRACHMAN DB, et al. Prednisone in Duchenne muscular dystrophy. The Lancet, 1974; 304: 1409-1412.

15. EUROPEAN MEDICINES AGENCY. Translarna: Ataluren. Science Medicines Health, 2020. Disponível em: http://www.ema.europa.eu/ema/index.jsp?curl=pages/medicines/human/medicines/002720/human_med_001742 .jsp\&mid=WC0b01ac058001d124. Acessado em: 16 de janeiro de 2021. 
16. FEDER D, LANGER AL. Uso dos corticóides no tratamento da distrofia muscular de Duchenne, 2005.

17. FONSECA JG, et al. Distrofia muscular de Duchenne: Complicações respiratórias e seu tratamento, 2007.

18. GARNELO L, et al. Acesso e cobertura da atenção primária à saúde para populações rurais e urbanas na região norte do Brasil. Saúde em Debate, 2018; 42: 1-99.

19. LEBEL DE, et al. Glucocorticoid treatment for the prevention of scoliosis in children with Duchenne muscular dystrophy: Long-term follow-up. The Journal of Bone and Joint Surgery-American, 2013; 95: 1057-1061.

20. MATTHEWS E, et al. Corticosteroids for the treatment of Duchenne muscular dystrophy cochrane database of systematic reviews, 2016.

21. MCDONALD CM, et al. Deflazacort vs prednisone treatment for Duchenne muscular dystrophy: A meta-analysis of disease progression rates in recent multicenter clinical trials. Muscle and Nerve, 2020; 61: 26-35.

22. MOXLEY RT, et al. Practice parameter: Corticosteroid treatment of Duchenne dystrophy - report of the quality standards subcommittee of the american academy of neurology and the practice committee of the child neurology society. National Library of Medicine, 2005; 1: 13-20.

23. NEGRONI E, et al. Stem cells and muscle diseases: Advances in cell therapy strategies. National Library of Medicine, 2015; 3: 270-287.

24. NORITZ G, et al. Primary care and emergency department management of the patient with Duchenne muscular dystrophy. Official Journal of the American Academy of Pediatrics, 2018; 142: S90-S98.

25. QUINLIVAN R. Early diagnosis of Duchenne muscular dystrophy is essential to improve long term outcomes. Archives of Disease in Childhood, 2014; 99: 1061.

26. SANTOS MA, et al. Distrofia muscular de Duchenne: Análise eletrocardiográfica de 131 pacientes. Arquivo Brasileiro de Cardiologia, 2010.

27. SOUZA IE, et al. Distrofia muscular de Duchenne: Complicações e tratamentos. Revista Fafibe On-Line, 2015; 1: 178187.

28. STREHLE EM, STRAUB V. Recent advances in the management of Duchenne muscular dystrophy. Archives of Disease in Childhood, 2015; 100: 1173-1177.

29. TAWALBEH S, et al. Comparison of serum pharmacodynamic biomarkers in prednisone-versus deflazacort-treated Duchenne muscular dystrophy boys. Journal of Personalized Medicine, 2020; 10: 164. 